\title{
CONCEPTUAL STUDY OF AN ULTRA-SHORT BUNCH LENGTH MONITOR
}

\author{
D. Alesini, M. Di Giosa, A. Mostacci, L. Palumbo
}

\section{Abstract}

A bunch length monitor for very short charge bunches based on microwave spectroscopy has been conceptually studied. It consists of a coaxial cavity coupled to the beam pipe through four symmetrical apertures. This signal excited by the bunch can be collected by a small antenna connected to a coaxial line transporting the signal to the measurement device. By frequency domain analysis of the measured signal it is possible to obtain the bunch lenght. The analytical frequency response relating the primary field radiated by the bunch in the beam pipe and the measurable electromagnetic field has been determined.

\section{INTRODUCTION}

A number of bunch lenght monitors exist either in the time domain either in the frequency domain. Time domain methods need a complex hardware, greately disturb the beam and have a low resolution. We particularly interest in microwave spectroscopy based on the analysis of the RF spectrum of the bunch. Different techniques have been proposed to couple the field radiated by the bunch in a transmission line leading the signal to the detection system.

Here we present a bunch length monitor based on a coaxial resonator coupled to the beam pipe through four symmetrical apertures.

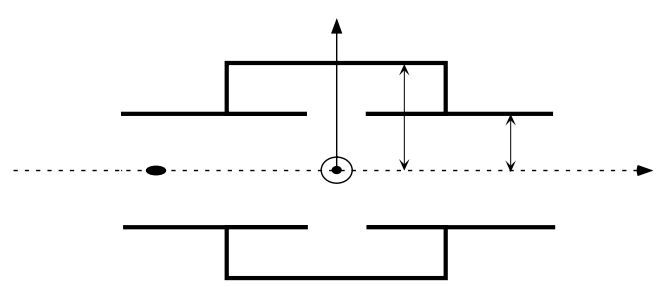

Figure 1: Structure geometry.

\section{DESCRIPTION OF THE METHOD}

According to the diffraction Bethe's theory [1] a hole in a perfectly conducting plane screen hitten by an electromagnetic field is equivalent to a combination of electric and magnetic dipoles. The electric and magnetic dipole momenta $\mathbf{P}$ and $\mathbf{M}$ are proportional to the normal electric field and the tangent magnetic field at the aperture respectively, through the electric and magnetic polarizability coefficients $\alpha_{e}$ and $\bar{\alpha}_{\mathbf{m}}$.

According to the modified Bethe's theory [2] the electric and magnetic dipoles are:

$$
\begin{aligned}
& \mathbf{P}=\mp \varepsilon \bar{\alpha}_{\mathbf{e}} \cdot\left[\mathbf{E}_{\mathbf{0 p}}+\mathbf{E}_{\mathbf{s p}}-\mathbf{E}_{\mathbf{s c}}\right] \\
& \mathbf{M}=\mp \bar{\alpha}_{\mathbf{m}} \cdot\left[\mathbf{H}_{\mathbf{0 p}}+\mathbf{H}_{\mathbf{s p}}-\mathbf{H}_{\mathbf{s c}}\right]
\end{aligned}
$$

where $\mathbf{E}_{\mathbf{0 p}}, \mathbf{H}_{\mathbf{0 p}}$ is the primary field radiated by the beam, $\mathbf{E}_{\mathrm{sp}}, \mathbf{H}_{\mathrm{sp}}$ is the field scattered in the circular waveguide and $\mathbf{E}_{\mathbf{s c}}, \mathbf{H}_{\mathbf{s c}}$ is the field in the coaxial cavity.

The scattered field in the circular waveguide can be expressed as a sum of modes propagating in both longitudinal directions:

$$
\begin{aligned}
\mathbf{E}_{\mathbf{s p}} & =\sum_{\mathbf{n}, \mathbf{m}}\left[\mathbf{a}_{\mathbf{n m}} \mathbf{E}_{\mathbf{s p}(\mathbf{n}, \mathbf{m})}^{+} \theta(\mathbf{z})+\mathbf{b}_{\mathbf{n m}} \mathbf{E}_{\mathbf{s p}(\mathbf{n}, \mathbf{m})}^{-} \theta(-\mathbf{z})\right] \\
\mathbf{H}_{\mathbf{s p}} & =\sum_{\mathbf{n}, \mathbf{m}}\left[\mathbf{a}_{\mathbf{n} \mathbf{m}} \mathbf{H}_{\mathbf{s p}(\mathbf{n}, \mathbf{m})}^{+} \theta(\mathbf{z})+\mathbf{b}_{\mathbf{n m}} \mathbf{H}_{\mathbf{s p}(\mathbf{n}, \mathbf{m})}^{-} \theta(-\mathbf{z})\right]
\end{aligned}
$$

where $\mathbf{E}_{\mathrm{sp}}^{ \pm}, \mathbf{H}_{\mathrm{sp}}^{ \pm}$are the modal functions and $\theta(z)$ is the Heaviside function. The expansion coefficients can be determined applying the Lorentz reciprocity principle which relates the fields to the electric and magnetic sources $\mathbf{J}_{\mathbf{e}}$, $\mathbf{J}_{\mathrm{m}}$ :

$$
\begin{aligned}
& a_{n m}=\frac{1}{2} j \omega\left(\mu \mathbf{H}_{\mathbf{s p}(\mathbf{n}, \mathbf{m})}^{-} \cdot \mathbf{M}-\mathbf{E}_{\mathbf{s p}(\mathbf{n}, \mathbf{m})}^{-} \cdot \mathbf{P}\right) \\
& b_{n m}=\frac{1}{2} j \omega\left(\mu \mathbf{H}_{\mathbf{s p}(\mathbf{n}, \mathbf{m})}^{+} \cdot \mathbf{M}-\mathbf{E}_{\mathbf{s p}(\mathbf{n}, \mathbf{m})}^{+} \cdot \mathbf{P}\right)
\end{aligned}
$$

The field excited in the cavity can be expanded in terms of complete orthonormal functions:

$$
\begin{aligned}
& \mathbf{E}=\sum_{\mathbf{n}} \mathbf{e}_{\mathbf{n}} \mathbf{E}_{\mathbf{n}} \\
& \mathbf{H}=\sum_{\mathbf{n}} \mathbf{h}_{\mathbf{n}} \mathbf{H}_{\mathbf{n}}
\end{aligned}
$$

where $\mathbf{E}_{\mathbf{n}}, \mathbf{H}_{\mathbf{n}}$ are the electric and magnetic solenoidal modes. The coefficients $e_{n}, h_{n}$ can be obtained manipulating the Maxwell equations [2].

Either the coefficients $a_{n m}, b_{n m}$ either the coefficients $e_{n}, h_{n}$ are function of electric and magnetic dipoles so substituting their expressions in the (1)-(2) we obtain a system for $\mathbf{P}$ and $\mathbf{M}$.

For the simmetry of the structure only quadrupolar modes propagate in the pipe so, assuming that the operating frequency is belowe the cut-off frequency of the first quadrupolar mode, there will not be propagating modes in the circular guide. The (1)-(2) then become:

$$
\begin{aligned}
& \mathbf{P}=\varepsilon \bar{\alpha}_{\mathbf{e}} \cdot\left[\mathbf{E}_{\mathbf{0 p}}-\mathbf{E}_{\mathbf{s c}}\right] \\
& \mathbf{M}=\bar{\alpha}_{\mathbf{m}} \cdot\left[\mathbf{H}_{\mathbf{0 p}}-\mathbf{H}_{\mathbf{s c}}\right]
\end{aligned}
$$


We just have to determine the field in the resonator and the corresponding coefficients $e_{n}, h_{n}$ :

$$
\begin{gathered}
e_{n}=\frac{-k_{n} \int_{V} j \omega \mu \mathbf{M} \cdot \mathbf{H}_{\mathbf{n}} \mathbf{d V}-\mathbf{j} \omega \mu \tilde{\mathbf{q}} \int_{\mathbf{V}} \mathbf{j} \omega \mathbf{P} \cdot \mathbf{E}_{\mathbf{n}} \mathbf{d V}}{\tilde{k}} \\
h_{n}=\frac{k_{n} \int_{V} j \omega \mathbf{P} \cdot \mathbf{E}_{\mathbf{n}} \mathbf{d} \mathbf{V}-\mathbf{j} \omega \varepsilon \int_{\mathbf{V}} \mathbf{j} \omega \mu \mathbf{M} \cdot \mathbf{H}_{\mathbf{n}} \mathbf{d V}}{\tilde{k}}
\end{gathered}
$$

where:

$$
\tilde{q}=1+\frac{1-j}{Q_{n}} ; \tilde{k}=k_{n}^{2}-k^{2} \tilde{q}
$$

and $Q_{n}$ is the quality factor of the cavity. Supposing only $T E M$ modes are resonating in the cavity at $\omega_{\ell}=\frac{\ell c \pi}{L}$ we find that the field excited in the cavity is:

$$
\begin{gathered}
E_{s c r}^{t o t}(r, \varphi, z)=4 \frac{k^{2} \tilde{q}}{\tilde{k}} \frac{\alpha_{e} E_{0 r} E_{\ell}}{1+4 \alpha_{e} \tilde{q} \frac{k^{2}}{\tilde{k}} E_{\ell}^{2}} E_{\ell}(r, \varphi, z) \\
H_{s c \varphi}^{t o t}(r, \varphi, z)=4 \frac{j \omega k_{\ell}}{\tilde{k}} \frac{\varepsilon \alpha_{e} E_{0 r} E_{\ell}}{1+4 \alpha_{e} \tilde{q} \frac{k^{2}}{\hat{k}} E_{\ell}^{2}} H_{\ell}(r, \varphi, z)
\end{gathered}
$$

for odd $\ell$ values, and:

$$
\begin{aligned}
& E_{s c r}^{t o t}(r, \varphi, z)=4 \frac{j \omega \mu k_{\ell}}{\tilde{k}} \frac{\alpha_{m \perp} H_{0 \varphi} H_{\ell}}{1-4 \alpha_{m \perp} \frac{k^{2}}{\tilde{k}} H_{\ell}^{2}} E_{\ell}(r, \varphi, z) \\
& H_{s c \varphi}^{t o t}(r, \varphi, z)=-4 \frac{k^{2}}{\tilde{k}} \frac{\alpha_{m \perp} H_{0 \varphi} H_{\ell}}{1-4 \alpha_{m \perp} \frac{k^{2}}{\tilde{k}} H_{\ell}^{2}} H_{\ell}(r, \varphi, z)
\end{aligned}
$$

for even $\ell$ values. Finally we achieve that the electric dipole excites frequencies that are odd multiple of the resonator fundamental frequency $\omega_{\ell}$ and the magnetic dipole excites even multiples.

The signal excited in the resonator can be collected introducing a small loop normal to the magnetic field lines. In order to have an efficient magnetic coupling the loop will be positioned where magnetic field is highest. If the loop is small compared with cavity dimensions, the magnetic field may be assumed constant over its area and the induced voltage is:

$$
V=j \omega \mu \bar{H} S_{l}
$$

where $S_{l}$ is the loop area and $H$ is the average magnetic field that in the vicinity of the $n$-th resonance is approximately given by $h_{n} H_{n}$. The signal will be finally transported by a coaxial cable to the measurement device. The analytical transfer function for a TEM mode resonating in the cavity is:

$$
\begin{aligned}
& V_{o \ell}=j \omega \mu S_{l} A_{\text {coax }} . \\
& \left\{\begin{array}{l}
-4 \frac{j \omega k_{\ell}}{\tilde{k}} \frac{1}{b \pi L \ln \left(\frac{d}{b}\right)} \frac{\varepsilon \alpha_{e} e^{-\gamma s} E_{0 r}(r=b, z=0)}{1+4 \alpha_{e} e^{-\gamma s} \tilde{q} \frac{k^{2}}{\tilde{k}} \frac{1}{b^{2} \pi L \ln \left(\frac{d}{b}\right)}} \frac{\sin \left(k_{\ell} z\right)}{r} \text { odd } \ell \\
4 \frac{k^{2}}{\tilde{k}} \frac{1}{b \pi L \ln \left(\frac{d}{b}\right)} \frac{\alpha_{m \perp} e^{-\beta s} H_{0 \varphi}(r=b, z=0)}{1-4 \alpha_{m \perp} e^{-\beta s} \frac{k^{2}}{\tilde{k}} \frac{1}{b^{2} \pi L \ln \left(\frac{d}{b}\right)}} \frac{\cos \left(k_{\ell} z\right)}{r} \text { even } \ell
\end{array}\right.
\end{aligned}
$$

where $A_{\text {coax }}$ is the coaxial cable attenuation.

\section{A NUMERICAL APPLICATION}

A coaxial cavity for measuring bunch lenght in the range $1-5 \mathrm{~mm}$ has been dimensioned for a circular pipe with $b=1 \mathrm{~cm}, d=2 \mathrm{~cm}$ and a cut-off frequency of $8.79 \mathrm{GHz}$. We considered only TEM modes resonating at $f_{i}=i f_{1}$, with $f_{1}=2.9 \mathrm{GHz}$ and $i=1, \ldots 5$; these frequencies are all below the cut-off frequency of the first quadrupolar mode $T E_{21}(14.58 \mathrm{GHz})$. The parameters of the rectangular apertures having the longest side in the longitudinal direction are reported in table 1 .

Table 1: Some cavity parameters

\begin{tabular}{|l|c|}
\hline$L$ & $5.2 \mathrm{~cm}$ \\
$W_{a}$ & $2 \mathrm{~mm}$ \\
$L_{a}$ & $5 \mathrm{~mm}$ \\
$\tilde{\alpha}_{e}$ & $-5.76 \cdot 10^{-10} \mathrm{~m}^{3}$ \\
$\tilde{\alpha}_{m}$ & $9.29 \cdot 10^{-10} \mathrm{~m}^{3}$ \\
\hline
\end{tabular}

Assuming $f_{1}$ as referring frequency we calculated the analytical expressions of detected powers ratios:

$$
\begin{aligned}
\frac{P_{i}}{P_{1}} & =i^{2}\left(\frac{Q_{i}}{Q_{1}}\right)^{2}\left|\frac{\tilde{\Lambda}\left(\omega_{i}\right)}{\tilde{\Lambda}\left(\omega_{1}\right)}\right|^{2} . \\
& \cdot \frac{\left(b^{2} \pi L \ln \left(\frac{d}{b}\right)-4 \tilde{\alpha}_{e} Q_{1}\right)^{2}+\left(b^{2} \pi L \ln \left(\frac{d}{b}\right)-4 \tilde{\alpha}_{e}\right)^{2}}{\left(b^{2} \pi L \ln \left(\frac{d}{b}\right)-4 \tilde{\alpha}_{e} Q_{i}\right)^{2}+\left(b^{2} \pi L \ln \left(\frac{d}{b}\right)-4 \tilde{\alpha}_{e}\right)^{2}}
\end{aligned}
$$

for odd $i$ values, and:

$$
\begin{aligned}
\frac{P_{i}}{P_{1}} & =i^{2}\left(\frac{Q_{i}}{Q_{1}}\right)^{2}\left(\frac{\tilde{\alpha}_{m}}{\tilde{\alpha}_{e}}\right)^{2}\left|\frac{\tilde{\Lambda}\left(\omega_{i}\right)}{\tilde{\Lambda}\left(\omega_{1}\right)}\right|^{2} . \\
& \frac{\left(b^{2} \pi L \ln \left(\frac{d}{b}\right)-4 \tilde{\alpha}_{e} Q_{1}\right)^{2}+\left(b^{2} \pi L \ln \left(\frac{d}{b}\right)-4 \tilde{\alpha}_{e}\right)^{2}}{\left(b^{2} \pi L \ln \left(\frac{d}{b}\right)+4 \tilde{\alpha}_{m} Q_{i}\right)^{2}+\left(b^{2} \pi L \ln \left(\frac{d}{b}\right)\right)^{2}}
\end{aligned}
$$

for even $i$ values. where $\tilde{\Lambda}\left(\omega_{i}\right)$ is the bunch spectrum [8]. The bunch length $\sigma_{z}$ in the range $1-5 \mathrm{~mm}$ can be obtained from the curves in figure 2 . We considered both cases of

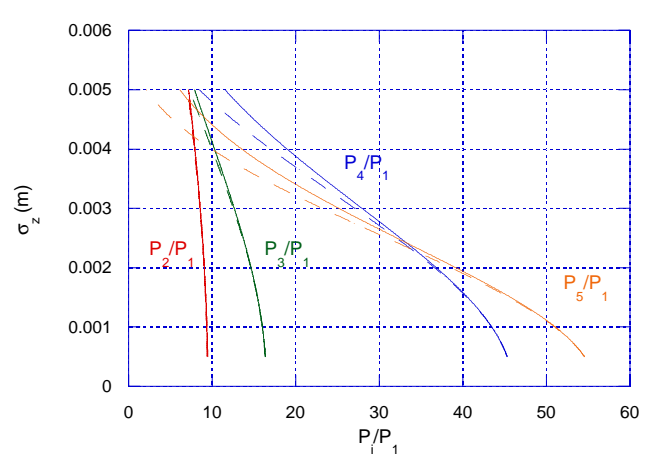

Figure 2: Dependence of bunch length on powers ratio.

a single bunch with gaussian (solid lines) and rectangular (dotted lines) distribution. It can be easily argued that the highest frequencies $f_{4}$ and $f_{5}$ can be used for measuring bunch lenghts in the range $1-5 \mathrm{~mm}$. 


\section{CONCLUSIONS}

We studied a new bunch lenght monitor consisting of a coaxial resonator coupled to the beam pipe through four symmetrical apertures. The system presented is simple to realize; it does not disturb the beam and it may be used for both linear and circular accelerators. The analytical transfer function has been calculated so that a calibration procedure is simplified. This method is also intended to determine transverse beam position by means of asymmetrical modes $T E, T M$.

\section{APPENDIX}

The resonant modes of a cavity can be determined from the modes propagating in a coaxial waveguide with the additional condition of null tangential electric field on the end plates. From these expressions it is possible to obtaine the coaxial resonator eigenfunctions [4]:

\section{$T E$ modes}

$$
\begin{aligned}
& \boldsymbol{E}_{n m \ell}=C_{n m \ell} \\
& {\left[-\frac{n}{r}\left[J_{n}\right]_{T E}\left\{\begin{array}{l}
-\sin (n \varphi) \\
\cos (n \varphi)
\end{array}\right\}\left\{\begin{array}{l}
\cos \left(k_{z \ell} z\right) \text { odd } \ell \\
\sin \left(k_{z} \ell\right) \text { even } \ell
\end{array}\right\} \hat{\boldsymbol{r}}+\right.} \\
& \left.+k_{t(n, m)}\left[J_{n}^{\prime}\right]_{T E}\left\{\begin{array}{l}
\cos (n \varphi) \\
\sin (n \varphi)
\end{array}\right\}\left\{\begin{array}{l}
\cos \left(k_{z \ell} z\right) \text { odd } \ell \\
\sin \left(k_{z} \ell\right) \text { even } \ell
\end{array}\right\} \hat{\varphi}\right] \\
& \boldsymbol{H}_{\boldsymbol{n} \boldsymbol{m} \ell}=C_{n m \ell} \frac{k_{z \ell}}{k_{n m \ell}} . \\
& {\left[-k_{t(n, m)}\left[J_{n}^{\prime}\right]_{T E}\left\{\begin{array}{l}
\cos (n \varphi) \\
\sin (n \varphi)
\end{array}\right\}\left\{\begin{array}{l}
-\sin \left(k_{z \ell} z\right) \text { odd } \ell \\
\cos \left(k_{z} \ell\right) \text { even } \ell
\end{array}\right\} \hat{\boldsymbol{r}}+\right.} \\
& -\frac{n}{r}\left[J_{n}\right]_{T E}\left\{\begin{array}{l}
-\sin (n \varphi) \\
\cos (n \varphi)
\end{array}\right\}\left\{\begin{array}{l}
-\sin \left(k_{z \ell} z\right) \text { odd } \ell \\
\cos \left(k_{z} \ell\right) \text { even } \ell
\end{array}\right\} \hat{\varphi}+ \\
& \left.-\frac{k_{t(n, m)}^{2}}{k_{z \ell}}\left[J_{n}\right]_{T E}\left\{\begin{array}{l}
\cos (n \varphi) \\
\sin (n \varphi)
\end{array}\right\}\left\{\begin{array}{l}
\cos \left(k_{z \ell} z\right) \text { odd } \ell \\
\sin \left(k_{z} \ell\right) \text { even } \ell
\end{array}\right\} \hat{\boldsymbol{z}}\right] \\
& T M \text { modes } \\
& \boldsymbol{E}_{n m \ell}=D_{n m \ell} \frac{k_{z \ell}}{k_{n m \ell}} . \\
& {\left[k_{t(n, m)}\left[J_{n}^{\prime}\right]_{T M}\left\{\begin{array}{l}
\cos (n \varphi) \\
\sin (n \varphi)
\end{array}\right\}\left\{\begin{array}{l}
\cos \left(k_{z \ell} z\right) \text { odd } \ell \\
-\sin \left(k_{z \ell} z\right) \text { even } \ell
\end{array}\right\} \hat{\boldsymbol{r}}+\right.} \\
& +\frac{n}{r}\left[J_{n}\right]_{T M}\left\{\begin{array}{l}
-\sin (n \varphi) \\
\cos (n \varphi)
\end{array}\right\}\left\{\begin{array}{l}
\cos \left(k_{z \ell} z\right) \text { odd } \ell \\
-\sin \left(k_{z \ell} z\right) \text { even } \ell
\end{array}\right\} \hat{\varphi}+ \\
& \left.+\frac{k_{t(n, m)}^{2}}{k_{z \ell}}\left[J_{n}\right]_{T M}\left\{\begin{array}{l}
\cos (n \varphi) \\
\sin (n \varphi)
\end{array}\right\}\left\{\begin{array}{l}
\sin \left(k_{z \ell} z\right) \text { odd } \ell \\
\cos \left(k_{z \ell} z\right) \text { even } \ell
\end{array}\right\} \hat{z}\right] \\
& \boldsymbol{H}_{\boldsymbol{n} \boldsymbol{\ell} \boldsymbol{\ell}}=D_{n m \ell} \\
& {\left[\frac{n}{r}\left[J_{n}\right]_{T M}\left\{\begin{array}{l}
-\sin (n \varphi) \\
\cos (n \varphi)
\end{array}\right\}\left\{\begin{array}{l}
\sin \left(k_{z \ell} z\right) \text { odd } \ell \\
\cos \left(k_{z \ell} z\right) \text { even } \ell
\end{array}\right\} \hat{\boldsymbol{r}}+\right.} \\
& \left.-k_{t(n, m)}\left[J_{n}^{\prime}\right]_{T M}\left\{\begin{array}{l}
\cos (n \varphi) \\
\sin (n \varphi)
\end{array}\right\}\left\{\begin{array}{l}
\sin \left(k_{z \ell} z\right) \text { odd } \ell \\
\cos \left(k_{z \ell} z\right) \text { even } \ell
\end{array}\right\} \hat{\varphi}\right]
\end{aligned}
$$

\section{$T E M$ modes}

$$
\begin{aligned}
\mathbf{E}_{\ell} & =\frac{\mathbf{A}_{\ell}}{\mathbf{r}}\left\{\begin{array}{c}
\cos \left(k_{\ell} z\right) \text { odd } \ell \\
-\sin \left(k_{\ell} z\right) \text { even } \ell
\end{array}\right\} \hat{\mathbf{r}} \\
\mathbf{H}_{\ell} & =-\frac{\mathbf{A}_{\ell}}{\mathbf{r}}\left\{\begin{array}{l}
\sin \left(k_{\ell} z\right) \text { odd } \ell \\
\cos \left(k_{\ell} z\right) \text { even } \ell
\end{array}\right\} \hat{\varphi}
\end{aligned}
$$

where:

$$
\begin{aligned}
& J_{n}\left(k_{t(n, m)} r\right)-\frac{J_{n}\left(k_{t(n, m)} b\right)}{Y_{n}\left(k_{t(n, m)} b\right)} Y_{n}\left(k_{t(n, m)} b\right)=\left[J_{n}\right]_{T M} \\
& J_{n}^{\prime}\left(k_{t(n, m)} r\right)-\frac{J_{n}\left(k_{t(n, m)} b\right)}{Y_{n}\left(k_{t(n, m)} b\right)} Y_{n}^{\prime}\left(k_{t(n, m)} r\right)=\left[J_{n}^{\prime}\right]_{T M}
\end{aligned}
$$

The normalization coefficients can be found from the conditions:

$$
\int_{V}\left|\mathbf{E}_{\mathbf{n m} \ell}\right|^{2} \mathbf{d} \mathbf{V}=\mathbf{1}
$$

\section{REFERENCES}

[1] H. A. Bethe, "Theory of diffraction by small holes", Phys. Rev., vol. 66, 1944

[2] R. E. Collin, "Field Theory of Guided Waves", IEEE Press, New York, 1991

[3] K. Kurokawa, "The expansion of electromagnetic fields in cavities", IRE Transactions on microwave theory and techniques, vol. MTT-6, 1958

[4] J. Van Bladel, "Electromagnetic Fields", A Summa Book, Hemisphere publishing corporation, Washinghton, 1985

[5] C. Martinez, "Determination of Longitudinal Electron Bunch Lenghts on Picosecond Time Scales", PhD Thesis, Universitat Politècnica De Catalunya, 1999

[6] S. De Santis and al., "Coupling impedance of a hole in a coaxial beam pipe", Phys. Rev. E, vol. 54, 1996

[7] M. Dohlus and al., "Design and Performance of Bunch Lenght Monitor in the Range of 1-5 mm", Internal Report, Deutsches Elektronen-Synchrotron DESY, Hamburg, Germany, 1998

[8] L. Palumbo and al., "Wake fields and impedance", CASCERN Accelerator School: Fifth Advanced Accelerator Physics Course, Rhodes, 1993 